\title{
Administrative rationality and coping strategies in shift work
}

\author{
Renato Rocha Lieber, ${ }^{\mathrm{a},},{ }^{*}$ Rodrigo Neiva Kvieska ${ }^{\mathrm{a}}$ and Maurício Cesar Delamaro ${ }^{\mathrm{a}}$ \\ ${ }^{a}$ Department of Industrial Engineering UNESP University Estadual Paulista - Av Dr Ariberto Pereira da Cunha \\ 333, 12516-410 Guaratinguetá SP,Brazil.
}

\begin{abstract}
Shift work (SW) can affect worker health and productivity. Working at night, workers often accumulate fatigue and are less productive. In Brazil, laws have been drafted aiming to reduce night work and rotating shift hours. In order to slash costs, companies have been looking for new arrangements to improve productivity under these conditions. The purpose of this study was to examine management changes and their outcomes in a large glass factory located in an industrial region of Brazil. The results show that the management, seeking equal productivity among shifts, focused its efforts mainly on distributing employee expertise. The arrangement resulted in 12 different groups that combine to serve three fixed shifts. A same shift can be served by more than one group, and the members of a same group share days off on different days. There was no statistically significant productivity difference among the three shifts. The on-site examination showed that part of the production was held by the workers and transferred to the next shift in order for them to be able to meet the management's performance rate requirements. The finding shows how a Brazilian cultural trait (resistance without conflict) is used to drive coping in SW.
\end{abstract}

Keywords: Productivity, Resistance strategy, solidarity, Culture, Brazil

\section{Introduction}

Shift work (SW) can affect worker health and productivity. To overcome adverse conditions, workers engender different coping strategies as a way to maintain employment. Night shifts may be particularly adverse, considering the role played by biological variations [10]. In Brazil, the law determines night shifts must be shorter, while the Federal Constitution imposes an even greater reduction to the shifts of workers who are exposed to rotation shifts, a fact that brought about the need for an additional group to cover 24 hours of operation [2]. Businesses, in turn, seek to cut costs and improve productivity. As night shifts generate additional labor costs and are associated with lower productivity [4], shift arrangements have gotten a lot of attention from management, which seeks to increase productivity from the same available manufacturing resources [5]. Administrative measures and new shift arrangements have been proposed with the immediate purpose of generating common productivity standards in the different shifts, regardless of the working hours. Training has been provided in an attempt to reconcile the management's needs with those of the workers involved [7]. The purpose of this paper was to show, through a pilot study, how shift workers adjust to streamlining proposals within the context of the Brazilian industry.

\section{Methods}

A semi-automatic industrial plant located in a Brazilian industrial region was chosen. The plant produces glass products under a rather unorthodox shift system. In the recent past, its industrial management made several changes to the organization seeking to achieve the same standards of productivity in all work schedules. As each shift is staffed by different groups concomitantly, and each group has a different number of members, who do not share the same rest dates, it was necessary to set a weighting criterion instead of only comparing the productivity rate attained by each shift. Thus, in addition to the number of workers who manned the shift, the number of days

\footnotetext{
*Corresponding author. E-mail: lieber@feg.unesp.br
} 
worked since each of them had their day off was figured in. The productivity achieved in 20 consecutive days among those with more and fewer hours worked was then compared, separating per shift. The productivity rates of the night group with the most nights worked was also compared with the morning group with the fewest prior days worked. A variance analysis with 1 and 2 repetition factors was used.

\section{Results}

Production is handled by 12 groups that combine daily to man three permanent shifts. A given shift may combine members of two or three different groups. The group combination for a same shift changes every 2 days and then repeats itself after four consecutive changes, so 12 different arrangements have been made to staff each permanent shift. The statistical analysis showed no significant difference in productivity rates, between shifts or between groups, even when compared to the expected extreme fatigue conditions. The average hourly productivity rate on the days examined was similar among shifts, increasing along the shift and declining abruptly at the end of the shift. In the first hour of work, high rates are followed by a decrease, rising again in the next hour, Figure 1. The in situ analysis of the study showed that, at each shift, part of the production was retained by the workers and transferred to the next shift.

\section{Discussion}

There are few studies relating productivity and SW. In general, productivity slumps 3 to $52 \%$ at night [5]. Although working conditions at night differ from daytime ones, the lack of sleep among night workers imposes a challenge on maintaining performance [4]. In the case being studied, semiautomatic operations help the attainment of the expected productivity rates. However, the machinery is very old (over 30 years) at the company that was studied. There is great demand for machine adjustments, and work is done under intense heat and noise. These facts drive rapid fatigue. With the aim of reducing costs, the company eliminated shift rotation. Fatigue accumulates with fixed shifts, particularly during the night one. Still, there were no productivity differences. To dilute its expertise, the company dissolved the traditional arrangement and created an array of combination groups. Although it could be expected that technical complications would be re- solved better, the measure did little to alter the technical profile since everyone has very similar experience. On the other hand, the analysis of the average hourly productivity rates showed that the process of retaining part of the production and transferring it to the next shift is common. Through a solidarity arrangement, all three shifts attain the same standard of productivity imposed by management.

The lack of cooperation among work shifts and the imbalance in terms of skills and support services, among other reasons, have been highlighted as the causes of the lower SW productivity [11]. Studies show, however, that intra-shift camaraderie with workmates takes place in different occupations, such as among offshore workers [14], nurses [12] and train drivers [13]. In such cases, solidarity among coworkers and supervisors improved coping in SW. In the case studied here, solidarity is not only intra-shift, rather also inter-shifts, showing a particular process of resistance to the management's requirements.

Different types of resistance in the workplace have been identified [8], and retaining part of the production, which is known as "kitty," "bank" or "stash," was interpreted as an expression of individuality [1]. However, structural interventions increase the workers' sense of shared group identity [6]. Organizing groups in a large number of irregular arrangements in an attempt to dilute expertise, allowed for greater integration among workers to deal with the issue that was common to all.

The productivity targets imposed by management are achieved in three shifts. However, a process of resistance was necessary to "rationalize the irrationality" of the management's requirements [3]. "Buffers" created by the workers themselves was the solution that was most compatible with the Brazilian cultural context where people "say yes, even wanting to say no" [9].

\section{Conclusions}

The findings show that culture is relevant not only in the definition of shift work arrangements, but also in the coping strategies, adjusted within aspects that are common to the local shared culture. Coping strategies can involve a complex process of workplace resistance without necessarily excluding the management-imposed service targets. Particular conditions arising from SW may favor this process on a large scale. 


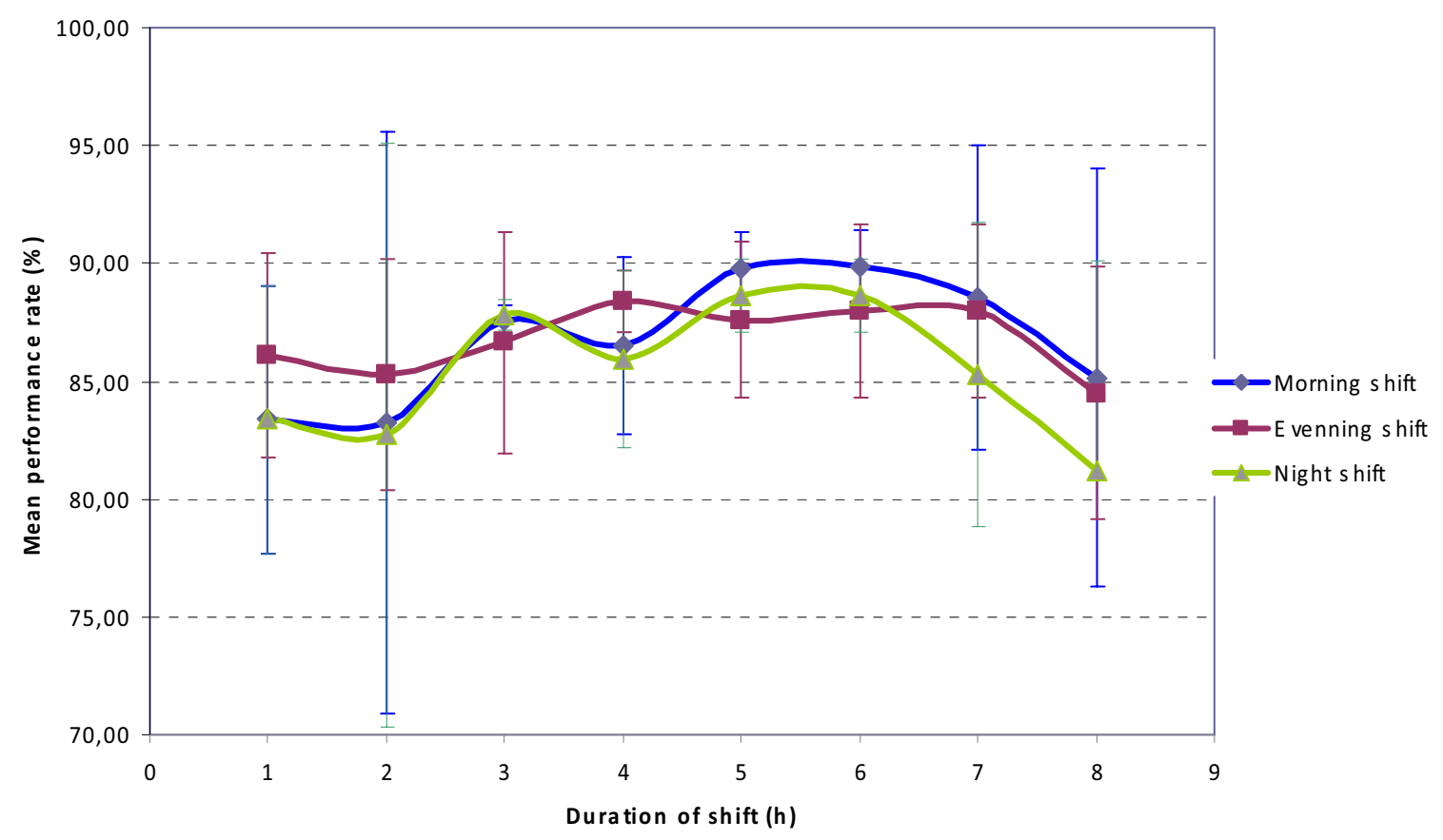

Fig.1. Variation of the mean performance rate according to the duration of each shift, calculated based on 20 consecutive days in three permanent shifts at a glass factory. The performance rate is calculated by dividing the number of parts produced by the machine's actual capacity (true capacity).

\section{References}

[1] D.L. Collinson, Strategies of resistance: power, knowledge and subjectivity in the workplace. In: Work and Society: A Reader, K. Grint, ed, Polity Press, Cambridge, 2000, pp. 163198.

[2] S. Demetriades and R. Pedersini, Working time in the EU and other global economies, Office for Official Publications of the European Communities, Luxembourg, 2008. (http://www.euro found.europa.eu/pubdocs/2008/65/en/1/EF0865EN.pdf)

[3] V.G. Devinatz, Manufacturing Resistance: Rationalizing the Irrationality of Managerial Control on the Shop Floor in a US Medical Electronics Factory, Employee Responsibilities and Rights Journal 19 (2007), 1-15.

[4] S. Folkard and P. Tucker, Shift work, safety and productivity, Occupational Medicine 53(2003), 95-101.

[5] A.S. Hanna, C. Chang, K. T. Sullivan and J. A. Lackney, Impact of shift work on labor productivity for labor intensive contractor, Journal of Construction Engineering and Management 131 (2008), 197-204.

[6] S.A. Haslam and S. Reicher, Stressing the group: social identity and the unfolding dynamics of responses to stress. $J$ Appl Psychol. 91(2006), 1037-52.

[7] A. Kerin and A. Aguirre, Improving health, safety, and profits in extended hours operations (shiftwork), Ind Health 43(2005), 201-8.
[8] D.K. Mumby, Theorizing Resistance in Organization Studies: A Dialectical Approach, Management Communication Quarterly 19(2005), 19-44.

[9] T. Novinger, Communicating with Brazilians: When "Yes" Means "No", University of Texas Press, Austin, 2003.

[10]A.K. Pati, A. Chandrawanshi and A. Reinberg, Shift work: Consequences and management, Current Science 81(2001), 32-52.

[11]D. Penkala, Improuving productivity and profitablility round the clock National Productivity Review 16(1997), 29-35.

[12]A. Pisarski, P. Bohle and V.J. Callan, Effects of coping strategies, social support and work-nonwork conflict on shift worker's health, Scand J Work Environ Health 24 (1998), Suppl 3, 141-5.

[13]S. Rainbird, K. Thompson and D. Dawson, The impact of organisational culture on fatigue management: The case of camaraderie amongst metropolitan train drivers. In: Living in a 24/7 world: The impact of circadian disruption on sleep, work and health, C. Sargent, D. Darwent and G.D. Roach, Eds, Australasian Chronobiology Society, Adelaide, Australia, 2010, pp. 29-33. (http://www.australasianchronobiology.org/ OrganisationalCulture.pdf)

[14]P. Ulleberg and T. Rundmo, Job stress, social support, job satisfaction and absenteeism among offshore oil personnel, Work \& Stress 11(1997), 215-228. 